\section{Antarctic astronomy in the clear}

SCIENCE at the South Pole has been conducted by a few sturdy individuals since the 1940s. In cosmic ray studies and astronomy, Martin Pomerantz was one of the first of these pioneers, so it was appropriate that the Bartol Institute, from which Pomerantz recently retired as director, was the host for a recent meeting* on what should be done to further astronomy at the South Pole. The meeting brought out many reasons, some obvious and some subtle, for battling the physical hardships and logistical difficulties of the Antarctic to make novel measurements.

For astronomers, the air over the South Pole has two special attributes which give it a unique transparency: the freezing cold which reduces the humidity to as little as 2 per cent, and the less well-known fact that the South Pole is more than 2,000 m above sea level, about the same as the Kitt Peak Observatory in Arizona. Together, these give the Antarctic atmosphere a transmission factor of 80 per cent in the millimetre range, where Mauna Kea in Hawaii, at close to $5,000 \mathrm{~m}$ altitude, can manage only $20-40$ per cent (F. Lo, Univ. Illinois).

Practical submillimetre astronomy, normally done from above the atmosphere, can be done from the South Pole, where the ground base offers the hope of building telescopes of several metres in diameter; the ASTRO project (Antarctic Submillimeter Telescope and Remote Observatory), a cooperation between AT\&T Bell Labs, Boston University and the University of Illinois, is a metre-class telescope designed to do far-infrared astronomy throughout the Antarctic winter (A. Stark, AT\&T). To be worth while, ASTRO must eventually offer an instrument much larger than the 1-m telescope aboard the Kuiper airborne observatory, a converted Boeing 747 .

The opportunities offered by this kind of astronomy, especially now that highefficiency CCD (charge-coupled device) detectors are available for infrared measurements, are wide-ranging. Infrared observations generally pick out cool, tenuous objects such as molecular clouds, and gas and dust being blown off by old stars or coming together as new ones. The large-scale, slow and complex astrophysical processes of galactic evolution show through more clearly in the infrared (R. Genzel, Max Planck Inst.; J. Peterson, Princeton Univ.).

At the other extreme, the transient and often controversial phenomena claimed by astronomers for whom $10^{11} \mathrm{eV}$ is the low end of their spectrum may best be studied from the South Pole. The advantage here lies not in any intrinsic property

* Astrophysics in Antarctica, Bartol Institute, University of Delaware, 8-10 June 1989. of the atmosphere, but in the fact that it stays in one place relative to the object observed (T. Weekes, Smithsonian Inst.).

In very high energy astronomy $\left(10^{11}\right.$ $10^{15} \mathrm{eV}$ ), an array of photodetectors on the ground is co-ordinated to look for a characteristic cone of Cerenkov radiation initiated by an energetic electron in the upper atmosphere; in ultra-high energy astronomy $\left(10^{15} \mathrm{eV}\right.$ and above $)$, particle detectors look for an 'air shower' of particles cascading from an initial collision between an energetic cosmic ray and an atmospheric atom. In both cases, the atmosphere is the detecting medium and the special value of observations at the South Pole is that a circumpolar celestial object is always seen through the same volume of atmosphere. The few real claims in this difficult branch of astronomy, such as the detection of periodic emission from known binary objects such as Cyg X-1, Her X-1 and some millisecond pulsars, are made less certain because the size and geometry of the region through which the instrument is looking changes with the Earth's rotation.

SPASE (South Pole Air Shower Experiment), an array of 14 scintillator detectors $30 \mathrm{~m}$ apart and situated $200 \mathrm{~m}$ from the South Pole (A. Hillas, Leeds Univ.) has recorded air showers throughout a southern winter. A bigger version is planned, but already it has demonstrated the feasibility of running a full-time experiment through the months of winter darkness.

More extravagantly, T. Stanev (Bartol Inst.) mentioned two versions of an instrument which uses ice, not air, as the detecting medium. In one, photomultiplier tubes would be equipped with heaters and allowed to fall several kilometres through the ice to rest in what is thought to be an expanse of pure, clear and bubble-free ice. There they would form a vast Cerenkov neutrino detector, like a bigger and frozen version of the Kamioka or IMB water-Cerenkov detectors that found the neutrinos from SN1987A. In a variation on this, Stanev described an idea due to the Russians Markov and Zheleznik for making a radio-Cerenkov detector in the ice. Neutrino-initiated electron-positron showers develop a charge asymmetry as they propagate; in ice they have a crosssection measured in metres. This moving small-scale charge emits radio waves, which could in principle be detected by microwave horns on the surface of the ice. Some experiments have been done at the Russian Vostok base, but the ice appears to be noisier than expected, and may prohibit a realistic detector of this sort.

David LIndley

David Lindley is an assistant editor at Nature.
A SUBTLE debate has been convulsing a sub-section of the feminist movement. Is it politically correct to use disposable babies' nappies, thus minimizing women's degrading unpaid household labour? Or are re-usable towelling nappies, saving the environment, ideologically more virtuous? Daedalus points out that many primitive peoples avoid this dilemma by using sphagnum moss as an absorbent for infantile excreta. The discarded moss re-enters the natural cycle, and may even survive and benefit from its fertilizing experience.

So Daedalus is devising the ultimate nappy. Drawing heavily on mustard-andcress flannel technology, it consists of an inert textile backing carrying a heavy growth of moss. After use it is simply laid aside; the fertilized moss soon metabolizes the excreta, producing more moss. It can then be used again. This cunning product solves the whole problem. It even grows most copiously in those areas most exposed to use, so that it shapes itself to its wearer's exact needs. A small initial supply of such truly 'green' nappies can be used and reused endlessly.

DREADCO textile technicians are now seeking to broaden this elegant principle. Some adult clothes (like sweat-bands and bandages) also exist primarily to absorb biological exudates. And all clothes get dirty, on the outside from environmental grime and on the inside from human perspiration and skin detritus. Living clothes which absorb dirt and build it into new fabric would brilliantly solve the twin nuisances of laundry and repair.

So DREADCO's biologists are seeding synthetic base-fabrics with various microfibrous plants to create a sort of biological corduroy. They are still debating whether small grasses, moss or algal filaments will be best, or whether the cloth can be constructed as a stable ecosystem of several mutually dependent species. Either way, the new living fabrics seem set to transform the fashion scene.

Self-cleaning, self-repairing and ecologically virtuous, 'Livewear' ${ }^{\circledR}$ will even keep its wearer clean by continuous gentle dermal scavenging. It will come in rather muted mossy and lichen-like colours, whose variegated pattern will slowly grow and change, especially at underarms, knees, elbows, and other moist or dirttrapping areas.

Livewear will also exercise a useful discipline on fashion fanatics. Regularly worn, a Livewear garment will be moistened and fertilized by its owner; wear and tear will be renewed and it will last indefinitely. But unworn, languishing in a dark, dry wardrobe, it will soon wilt and die. Surplus garments will automatically weed themselves out, while the timeless favourites live on and flourish.

David Jones 